\title{
Significance of Non-Antimicrobial Prevention of Urinary Tract Infection among Women: Future Perspective
}

\author{
Belal Ahmed Ibne Mahmood ${ }^{1}$
}

\author{
${ }^{1}$ Professor \& Head, Department of Microbiology, North-East Medical College, South Surma, Sylhet, Bangladesh; Email: \\ belalm1958@gmail.com
}

Urinary tract infection (UTI) is a very common disease among women. It has been estimated that about 50 to $60.0 \%$ of women will get at least one urinary tract infection in their lifetime $^{1}$. In uncomplicated UTI the infection is not associated with structural or functional disorders of the urinary tract. After this uncomplicated UTI around $25 \%$ of women experience a recurrent infection within 6 to 12 months, and around 5.0\% have several episodes within a year ${ }^{2}$. Recurrent UTIs are mostly occurred in young women; however, postmenopausal women are also often affected ${ }^{3}$. It has been found that about 75 to $85 \%$ of uncomplicated UTIs are caused by Escherichia coli ${ }^{4}$. According to the classic understanding of pathogenesis, almost all recurrent UTIs are ascending re-infections by the same clone or a different clone from the rectal reservoir ${ }^{5}$. However, an alternative pathway has emerged. In murine cystitis, uropathogenic $E$ coli binds to, invades, and replicate within the bladder urothelium to form intracellular bacterial communities, which dissociate and ultimately establish intracellular reservoirs that can seed recurrent UTIs $^{6}$. The presence of exfoliated intracellular bacterial communities and filamentous bacteria in the urine of women with acute cystitis suggests that the same intracellular bacterial community pathogenic pathway could have a role in human beings. These findings support the occurrence of an intracellular bacterial niche in some women with cystitis that could have important implications for recurrence and treatment of UTIs ${ }^{7}$. According to international guidelines, prevention of recurrent UTIs includes counselling and behavioural modifications followed by non-antimicrobial measures; antimicrobial prophylaxis is indicated only when nonantimicrobial measures are ineffective ${ }^{5}$.
For the prevention of UTI many non-antimicrobial measures have been tested like local hormonal support in postmenopausal women, cranberry products, probiotics, immunoactive prophylaxis, dmannose, endovesical instillation of hyaluronic acid and chondroitin sulphate ${ }^{8}$. Evidence for most of these interventions is low or supported only by results from small prospective randomised clinical trials. Immunoprophylaxis with UroVaxom is the only intervention that has grade $\mathrm{B}$ recommendation because it was more efficacious than placebo in several randomised placebo-controlled trials ${ }^{8}$. Although UroVaxom is made from an extract of heat-killed uropathogenic $E$. coli and thus can stimulate an improved immune response at infected sites, such as the urinary tract, it cannot be considered a vaccine against infections exclusively caused by uropathogenic $E$ coli. Development of a specific vaccine against extra-intestinal pathogenic $E$ coli, including uropathogenic $E$ coli, would offer another non-antimicrobial option for prevention of recurrent UTIs, which could provide a great clinical advantage.

Huttner et $\mathrm{al}^{9}$ have tested a bioconjugate vaccine containing the O-antigens of four $E$ coli serotypes. This tetravalent vaccine candidate was well tolerated and elicited functional antibody responses against all vaccine serotypes. The results of a metaanalysis ${ }^{10}$ of five randomised controlled trials of UroVaxom showed that the difference between patients without UTI during the follow-up period was about $20 \%$ higher in those treated with UroVaxom than in those given placebo and the mean number of recurrent UTI episodes was reduced by about $40 \%$ in the UroVaxom group. The duration of frequency was significantly shorter in 
the vaccine group than in the placebo group. However, Urovac ${ }^{\circledR}$ is a vaginal vaccine that contains 10 heat-killed uropathogenic bacterial species, including six different serotypes of uropathogenic Escherichia coli, Proteus vulgaris, Klebsiella pneumoniae, Morganella morganii and Enterococcus faecalis ${ }^{11}$. This vaccine induces primarily immunoglobulin $\mathrm{G}$ and immunoglobulin $\mathrm{A}$ in the urogenital tract, thereby reducing potential colonization of the vagina and bladder with uropathogens ${ }^{12}$. In three trials, all by the same group of investigators, placebo was compared with primary immunization or with primary immunization with booster immunizations ${ }^{13-14}$. Primary immunization consisted of three vaginal vaccine suppositories at weekly intervals. Booster immunization consisted of three additional vaccine suppositories at monthly intervals. Primary immunization alone did not reduce UTI recurrence. However, following the booster immunizations there was a prolonged time to the first recurrence of UTI, compared to primary immunization only or placebo.

However, conclusions about the vaccine's efficacy in preventing more invasive $E$. coli infections, such as bacteraemic infections, cannot be drawn on the basis of this finding, because bacterial count alone is not a factor for severity in uncomplicated UTIs ${ }^{15}$. Nonetheless, the preliminary results for this vaccine seem promising, and this trial is the first proof of concept in human beings that a vaccine might be effective against $E$ coli infections. Further studies of different doses and formulations of the candidate vaccine, and of the vaccine's efficacy against invasive infections, are recommended.

[Bangladesh Journal of Infectious Diseases 2016;3(2):27-28]

[How to cite this paper: Mahmood BAI. Significance of NonAntimicrobial Prevention of Urinary Tract Infection among Women: Future Perspective. Bangladesh Journal of Infectious Diseases 2016;3(2):27-28]

\section{References}

1. Foxman B, Brown P. Epidemiology of urinary tract infections: transmission and risk factors, incidence, and costs. Infectious Disease Clinics of North America 2003;17(2):227-41
2. Stamey TA. Recurrent urinary tract infections in female patients: an overview of management and treatment. Rev Infect Dis 1987; 9 (suppl 2): S195-210

3. Suskind AM, Saigal CS, Hanley JM, et al. Incidence and management of uncomplicated recurrent urinary tract infections in a national sample of women in the United States. Urology 2016; 90: 505

4. Naber KG, Schito G, Botto H, Palou J, Mazzei T. Surveillance study in Europe and Brazil on clinical aspects and antimicrobial resistance epidemiology in females with cystitis (ARESC): implications for empiric therapy. Eur Urol 2008; 54: 1164-1178

5. Lina TT, Khajanchi BK, Azmi IJ, Islam MA, Mahmood B, Akter M, et al. Phenotypic and Molecular Characterization of Extended-Spectrum Beta-LactamaseProducing Escherichia coli in Bangladesh. PLoS ONE 2014;9(10): e108735

6. Mulvey MA, Schilling JD, Hultgren SJ. Establishment of a persistent Escherichia coli reservoir during the acute phase of a bladder infection. Infect Immun 2001; 69: 4572-79

7. Rosen DA, Hooton TM, Stamm WE, Humphrey PA, Hultgren SJ. Detection of intracellular bacterial communities in human urinary tract infection. PLoS Med 2007;4: 1949-58

8. Grabe M, Bartoletti, Bjerklund-Johansen TE, et al. European Association of Urology guidelines on urological infections. http://uroweb.org/wp-content/uploads/EAUGuidelines-Urological-Infections-v2.pdf (accessed Feb 2, 2017).

9. Huttner A, Hatz C, van den Dobbelsteen G, et al. Safety, immunogenicity, and preliminary clinical efficacy of a vaccine against extra-intestinal pathogenic Escherichia coli in women with a history of recurrent urinary tract infection: a randomised, placebo-controlled trial. Lancet Infect Dis 2017; published online Feb 23. http://dx.doi.org/10.1016/S1473-3099(17)30108-1

10. Naber KG, Cho Y-H, Matsumoto T, Schaeff er AJ. Immunoactive prophylaxis of recurrent urinary tract infections: a meta-analysis. Int J Antimicr Agents 2009; 33: $111-19$

11. Beerepoot M, Geerlings S. Non-antibiotic prophylaxis for urinary tract infections. Pathogens. 2016;5(2):36

12. Hopkins WJ, Elkahwaji J, Beierle LM, Leverson GE, Uehling DT. Vaginal mucosal vaccine for recurrent urinary tract infections in women: Results of a phase 2 clinical trial. J Urol 2007;177:1349-1353

13. Uehling DT, Hopkins WJ, Elkahwaji JE, Schmidt DM, Leverson GE. Phase 2 clinical trial of a vaginal mucosal vaccine for urinary tract infections. The Journal of urology. 2003;170(3):867-9.

14. Uehling DT, Hopkins WJ, Beierle LM, Kryger JV, Heisey DM. Vaginal mucosal immunization for recurrent urinary tract infection: extended phase II clinical trial. The Journal of infectious diseases. 2001;183(Supplement_1):S81-3

15. Hooton T, Roberts P, Cox M, Stapleton A. Voided midstream urine culture and acute cystitis in premenopausal women. N Engl J Med 2013;369: 1883-91 